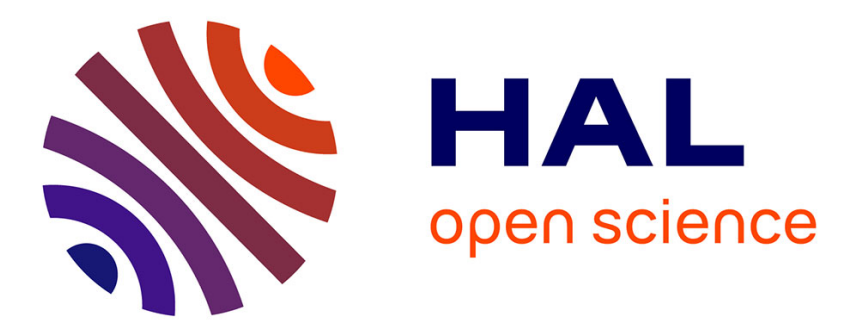

\title{
Tunable ultra-fast infrared generation in a gas-filled hollow core capillary by a four-wave mixing process
}

Olivia Zurita-Miranda, Coralie Fourcade-Dutin, Frederic Fauquet, Frederic

Darracq, Jean-Paul Guillet, Patrick Mounaix, Herve Maillotte, Damien

Bigourd

\section{To cite this version:}

Olivia Zurita-Miranda, Coralie Fourcade-Dutin, Frederic Fauquet, Frederic Darracq, Jean-Paul Guillet, et al.. Tunable ultra-fast infrared generation in a gas-filled hollow core capillary by a four-wave mixing process. Journal of the Optical Society of America B, 2022, 39 (3), pp.662. 10.1364/JOSAB.444574 . hal-03558771

\section{HAL Id: hal-03558771 \\ https://hal.science/hal-03558771}

Submitted on 4 Feb 2022

HAL is a multi-disciplinary open access archive for the deposit and dissemination of scientific research documents, whether they are published or not. The documents may come from teaching and research institutions in France or abroad, or from public or private research centers.
L'archive ouverte pluridisciplinaire HAL, est destinée au dépôt et à la diffusion de documents scientifiques de niveau recherche, publiés ou non, émanant des établissements d'enseignement et de recherche français ou étrangers, des laboratoires publics ou privés. 


\title{
Tunable ultra-fast infrared generation in a gas-filled hollow core capillary by a four-wave mixing process.
}

\author{
Olivia Zurita-Miranda, ${ }^{1,2,}$ * CORAlie FourCADE-Dutin, ${ }^{1}$ \\ Frederic Fauquet, ${ }^{1}$ Frederic DARracQ, ${ }^{1}$ JeAn-PAul Guillet, ${ }^{1}$ \\ Patrick Mounaix, ${ }^{1}$ Herve Maillotte, ${ }^{2}$ Damien Bigourd ${ }^{1 * * *}$
}

1 Laboratoire IMS, UMR CNRS 5218, Université de Bordeaux, 33400 Talence, France;

2 Département d'Optique, Institut FEMTO-ST, UMR CNRS 6174, Université Bourgogne Franche-Comté, 25030 Besançon, France

*Corresponding author:olivia.zurita-miranda@u-bordeaux.fr
**Corresponding author:damien.bigourd@u-bordeaux.fr

Received XX Month XXXX; revised XX Month, XXXX; accepted XX Month XXXX; posted XX Month XXXX (Doc. ID XXXXX); published XX Month XXXX

In this manuscript, we numerically and experimentally investigated the four-wave mixing process in a gas filled hollowcore capillary in the femtosecond regime. The interaction between a visible broad-band continuum and a chirped pump pulse resulted to the generation of a tunable near infrared pulse of 1.2 to $1.5 \mu \mathrm{m}$, and with the potentiality to reach the mid-infrared range. Numerical simulations were performed in order to fully understand the role of key parameters such as the gas pressure, chirps and relative delays of the involved pulses. The experimentation which demonstrated and highlighted the feasibility of the tunable femtosecond source, led to the generation of an idler at $1.2 \mu \mathrm{m}$ with a duration of $\sim 220 \mathrm{fs}$ at the direct output of the capillary. The duration can ultimately be reduced to 45 fs in the presence of phase compensators.

\section{INTRODUCTIONS}

Ultra-fast optical parametric amplification (OPA) is a popularly well-established method in a large range of configurations to obtain interesting properties such as a very wide-tunability, an ultra-broad gain-bandwidth for ultra-short pulses and a very-high temporal contrast [1,2]. The OPA process was performed in different materials, mainly by exploiting the second-order susceptibility of bulk non-linear crystals, but also in isotropic media where their third-order susceptibility is utilized [3]. In both cases, the interaction geometry is essential to get the optimal phase matching that allows efficient energy conversion from the pump to the targeted signal. To support the nonlinear process at very high power, the amplifier size can also be adjusted to decrease the pump intensity. Similarly, pulses are stretched to decrease the peak power at the system input while the targeted pulse is compressed after the amplification. This scheme, known as optical parametric chirped pulse amplification, guarantees the possibility of attaining a very high energy and/or high peak power [4].

An alternative procedure for ultra-fast OPA is to achieve the process in guided systems due to the significant advantages of the guiding geometry. Besides its robustness and compactness, the optical interaction between the involved pulses is more efficient thanks to the beam collinearity, excellent spatial beam profile and superposition that can be directly delivered within the waveguide. Particularly, we investigated this process in single-mode solid-core photonic crystal fibers in which the generation and the amplification were achieved from a Four Wave Mixing (FWM) mechanism. In this case, the phase matching is given by the nonlinear phase of the pump and the overall (chromatic and geometric) dispersion of the fiber [5].

As previously explained in bulk materials, the power scalability can be performed by increasing the fiber core diameter while keeping the suitable dispersion profile [6,7] or by stretching the involved pulses [8-10]. Another method is to perform the OPA process in a gas-filled waveguide in which the optical damage threshold is much higher than in silica. In this case, the dispersion can be controlled by the pressure in real time, allowing a tunable phase-matched process.

Few groups focused their research on the amplification in gas filled hollow-core capillaries (HCC), with a large core, in the visible [11], ultra-violet [12,13] and infrared ranges [14-16]. In most cases, the 
amplification was done with a multimodal phase matching in the HCC to amplify a targeted spectral range $[14,15]$. It is noteworthy that some investigations were performed in gas filled hollow-core photonic crystal fibers [17-20] but usually with limited experimental results obtained with a large fiber-core.

This work gathers analytical, numerical and experimental results to understand the FWM in HCC with a large core and to highlight the role of key parameters, as the signal and pump chirps. This detailed investigation will be of prime interest to design ultra-fast amplifiers. In this manuscript,-we focus on the generation of tunable idler pulses rather than on the amplification of the signal. Experimental results are also presented to highlight the feasibility of the techniques in comparison with the simulations.

\section{THEORETICAL BACKGROUND}

The general principle of a degenerated FWM relies on the interaction of a strong pump pulse (p), a weak injected signal (s), and a generated idler (i) in a material exhibiting a third order nonlinearity $\chi^{(3)}$. For an HCC system based on a FWM process, the nonlinearity arises from the gas confined in the waveguide. The pump and signal pulses are coupled into the HCC and a third wave (the idler) is generated during the propagation in the gas. The energy is transferred to the idler and signal from the pump under specific conditions depending on parameters such as the gas pressure, gas species, capillary properties and pump features. In order to enhance the energy conversion efficiency and induce a spectral tunability, the pump and the signal pulses will be chirped. Thus, different spectral components from the two pulses will temporally overlap. To quantify the contribution of all these parameters, we first look at the key ingredients required for the FWM and introduce known equations that are useful for the discussion.

\section{A. Phase-matching and gain}

To achieve an efficient FWM process, one of the key requirements is that all the involved waves satisfyies a phase-matching condition $\kappa$, which is maintained over a specific spectral bandwidth

$$
\kappa=\Delta \beta+\Delta \beta_{N L}
$$

where $\Delta \beta$ is the linear phase related to the gas pressure and waveguide properties. It is calculated from the sum of the propagation constant $\beta$ of all the waves; i.e., $\Delta \beta=\beta_{\mathrm{s}}+\beta_{\mathrm{i}}-2 \beta_{\mathrm{p}}$.

$\Delta \beta_{\mathrm{NL}}$ is the nonlinear phase induced from the pump pulse. Assuming no pump depletion, the expression of this nonlinear term is given by

$$
\Delta \beta_{N L}=2 \cdot \frac{\omega_{p} \cdot n_{2}}{A_{e f f} \cdot c} \cdot P_{P}
$$

with $\omega_{\mathrm{p}}$ the angular frequency at the pump wavelength, $\mathrm{c}$ the light speed, $A_{\text {eff }}$ the effective mode area and $\mathrm{n}_{2}$ the gas nonlinear index, which depends on the species and the pressure, $\mathrm{P}_{\mathrm{p}}$ the pump power. The second requirement is directly linked to the photon energy conservation law; i.e $2 \omega_{\mathrm{p}}=\omega_{\mathrm{s}}+\omega_{\mathrm{i}}$, where $\omega_{\mathrm{s}}$ and $\omega_{\mathrm{i}}$ are the angular frequencies of the signal and idler respectively. This means the signal and idler waves are symmetrically positioned around the pump; i.e $\omega_{\mathrm{s}}-\omega_{\mathrm{p}}=\omega_{\mathrm{p}}-\omega_{\mathrm{i}}$. Expanding each propagation constant $\beta$ in a Taylor series around $\omega_{\mathrm{p}}$, one can demonstrate that odd terms (mostly the third order dispersion term at the pump frequency) have no influence on the phase matching for a continuous pump wave [21].

In the undepleted regime and for a monochromatic pump wave, the FWM is well analytically described to understand the behavior of the process $[22,23]$. When only a single weak wave is injected in the HCC with the power $\mathrm{P}_{\mathrm{s}}(0)$, the power $\mathrm{P}_{\mathrm{s}}$, can be expressed as a function of the HCC length in an ingenuous form as :

$$
P_{s}(L)=P_{s}(0) \cdot\left[1+\left(\frac{\omega_{p} \cdot n_{2} \cdot P_{p}}{A_{e f f} \cdot c \cdot g} \cdot \sinh (g \cdot L)\right)^{2}\right]
$$

With parametric gain $g$ given by:

$$
g^{2}=\left[\left(\frac{\omega_{p} \cdot n_{2}}{A_{\text {eff }} \cdot c} \cdot P_{P}\right)^{2}-\left(\frac{\kappa}{2}\right)^{2}\right]
$$

From Eq. 3 and 4, we can estimate the output power of the signal in the undepleted regime given that $\mathrm{P}_{\mathrm{s}}$ is much weaker than $\mathrm{P}_{\mathrm{p}}$.

\section{B. Specific parameter for FWM in HCC}

The group-velocity-dispersion $\beta_{2}$ of the HCC is an important parameter required to tune the phase matching condition of the FWM (Eq. 1). It mostly depends on the waveguide core radius $(\phi)$, and refractive index of the gas, $n_{\text {gas }}$, which changes with the pressure $\mathrm{P}_{\text {gas }}$ and its species. $\mathrm{n}_{\text {gas }}$ can be calculated from the Sellmeier equation [24]. The modal propagation constant of a gas filled HCC is analytically calculated from the Marcatili and Schmelzer's model [25] as a function of the wavelength,

$$
\beta_{m, n}\left(\lambda, P_{\text {gas }}\right)=\left(\frac{2 \pi}{\lambda}\right) \sqrt{\mathbf{n}_{\text {gas }}{ }^{2}\left(\lambda, P_{\text {gas }}\right)-\frac{u_{m, n}{ }^{2} \lambda^{2}}{4 \pi^{2} \phi^{2}}}
$$

$\mathrm{u}_{\mathrm{m}, \mathrm{n}}$ is the $\mathrm{n}^{\text {th }}$ zero of the $\mathrm{m}^{\text {th }}$-order Bessel function of the first kind. For example, $\mathrm{u}_{11}=2.404$ for the fundamental mode $\mathrm{HE}_{11}$. From Eq. $5, \beta_{2}$ can be calculated for HE 11 and Fig. 1.a shows typical profiles when a HCC is filled with Argon for a core diameter of $150 \mu \mathrm{m}$. When the pressure increases, the dispersion curve is modified and the Zero Dispersion Wavelength (ZDW, $\beta_{2}=0 \mathrm{ps}^{2} / \mathrm{m}$ ) shifts to a longer wavelength [26]. The ZDW also changes with the core radius and the type of gas (Fig. 1.b). For example, the ZDW is set to a longer wavelength for xenon gas instead of argon for a constant core size and a fixed pressure of 1 bar. In all cases, the ZDW shifts to a lower wavelength as the core diameter decreases.

The value and the sign of $\beta_{2}$ are very important to predict and tune the FWM bands since it directly affects the phase-matching (Eq.1). In particular, when the pump lies in the normal dispersion regime, the FWM spectral lobes can be considerably shifted away from the pump wavelength, but typically at the expense of the spectral bandwidth [27]. Therefore, it is highly desirable to adjust the ZDW by changing not only the pressure, (Fig. 1.a) but also the core radius of the HCC and gas species (Fig.1.b). However, the power is also one important constraint when choosing the size of the HCC. For example, to avoid any damage of the cladding or some spurious nonlinearities in the HCC, a large core radius is preferably chosen. Therefore we decided to take a radius $\phi_{\mathrm{x}}$ of $75 \mu \mathrm{m}$ to fit with our experimental situation dealing with ultra-short pulse delivered by a 
Ti:Sa chain (800nm, 100fs, $1 \mathrm{~mJ}$ ) to avoid optical damage or spurious nonlinearity.

From Eq. 1-5, the gain, i.e $G=P_{s}(L) / P_{s}(0)$, is calculated (Fig. 1.c) for a pump continuous wave at $800 \mathrm{~nm}$ with a peak power of $1.6 \mathrm{GW}$ for several argon pressures. The spectral gain $\mathrm{G}$ curve is plotted as a function of the frequency offset from the pump; i.e $\omega_{s}-\omega_{p}$. In these cases, $\mathrm{n}_{2}=8.45 \times 10^{-24} \mathrm{~m}^{2} \mathrm{~W}^{-1}$ at 1 bar, and $\mathrm{A}_{\mathrm{eff}}=1.5 . \phi_{\mathrm{x}}{ }^{2}$. Clearly, the phase matching is tuned from the visible to the near infrared with a gain up to $15 \mathrm{~dB}$, and the spectrum shifts away from the pump wavelength when the pressure increases (Fig. 1.c). It should be noted that, at this pressure range, the FWM process is only phasematched when all the waves propagate in the fundamental mode.
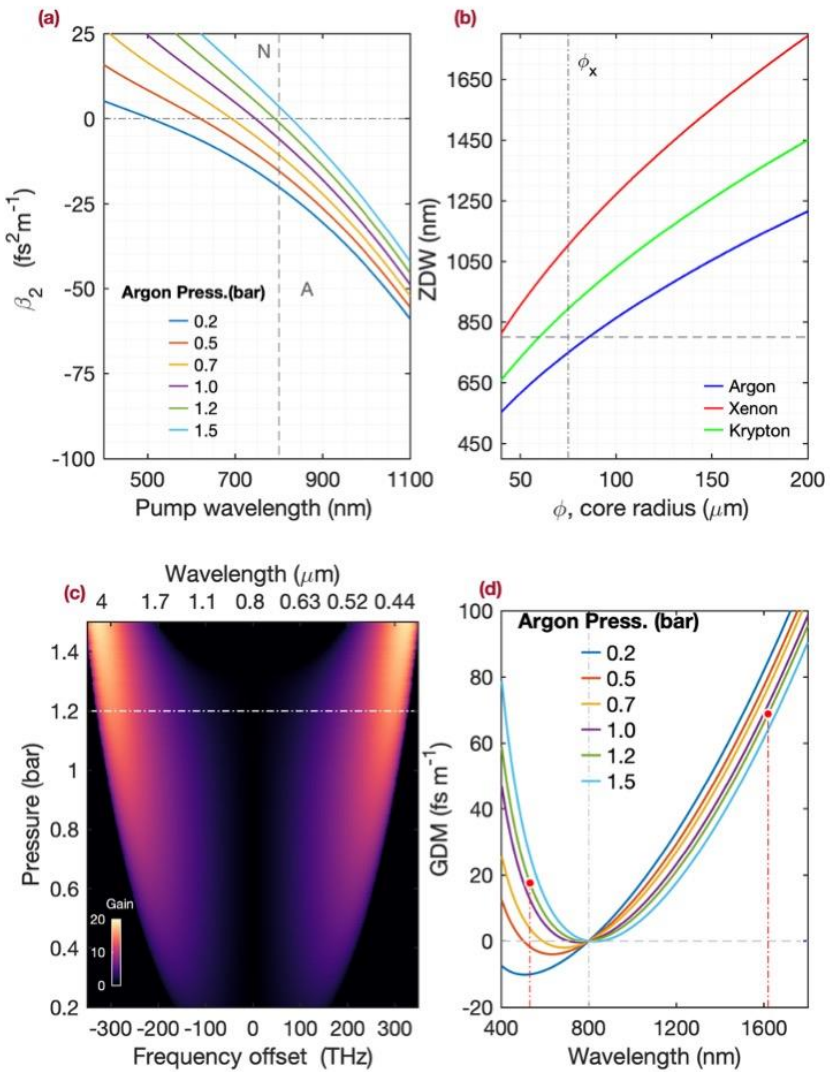

Fig .1 a) Dispersion curve of the HCC with a $150 \mu \mathrm{m}$ core diameter and filled with argon at different pressure b) ZDW as a function of the core radius at a pressure of 1 bar for three gas species c) Gain spectrum as a function of the gas pressure for a pump power of $1.6 \mathrm{GW}$. The white line depicts a reference pressure for Fig.2, and Fig. 3. d) Group delay mismatch in a HCC with a core diameter of $150 \mu \mathrm{m}$ and filled with different argon pressures. The red points are examples cited in the text.

\section{Specific parameters for ultra-fast FWM-OPA in HCC}

Since the involved pulses have an ultra-short duration in the range of $100 \mathrm{fs}$, the group delay mismatch (GDM) between the pump and the signal/idler is firstly calculated for an argon filled HCC with $\phi_{x}$ $=75 \mu \mathrm{m}$ (Fig. 1.d). As expected from the low refractive index, the GDM is relatively low for the gas pressure range. As an illustration, by considering pump and signal pulses whose central wavelengths are $800 \mathrm{~nm}$ and $530 \mathrm{~nm}$ respectively, the two pulses separate from each other at a rate $\sim 17.7 \mathrm{fs} . \mathrm{m}^{-1}$ for a pressure around 1.2 bar. This means the pump and signal pulses with a duration of $100 \mathrm{fs}$ do not overlap after propagation of $\sim 5.6 \mathrm{~m}$ (walk-offlength, LwL). Similarly, the corresponding idler at $\sim 1630 \mathrm{~nm}$ (Fig. 1.c), separates from the pump at a rate of $\sim 69 \mathrm{fs} . \mathrm{m}^{-1}$, corresponding to $\mathrm{LwL} \sim 1.4 \mathrm{~m}$ for a pulse duration of $100 \mathrm{fs}$.

Besides the GDM, spectral bandwidth is an important factor to consider in an ultra-fast amplifier. Indeed, as we aim to amplify a broadband signal, the phase-matched process should occur over a large spectral range [9] by taking benefit from the pump bandwidth. The previously described analytical model (section 2.A) does not fully explain the ultra-fast FWM since it does not include the contribution of the pump bandwidth. Each pump spectral component impacts the FWM process depending on the odd terms of the dispersion $[21,28]$. A qualitative approach is to calculate the coherence length $\mathrm{CL}=1 / \kappa$, from a tunable continuous pump wave (Fig. 2.a) with a wavelength scanned across the bandwidth of the broadband pump (e.g 750-850 nm). As an example, Figure. 2.b displays a selection of signal bands at a pressure of 0.75 bar for several pump central wavelengths.

The spectral bandwidth of the FWM increases when the broadband pump is used in comparison with the case where the pump is monochromatic. For example, in the two dimensional representation of the CL the FWM idler band expands from 1200 up to $2000 \mathrm{~nm}$ for the broadband pulse at a pressure of $0.75 \mathrm{bar}$, (line with grey shadow in Fig 2a).

At higher pressure, the FWM bands also became narrower and were shifted away from the pump spectrum since the pump lies in the normal dispersion for a pressure higher than 1.2 bar (Fig. 1.a).

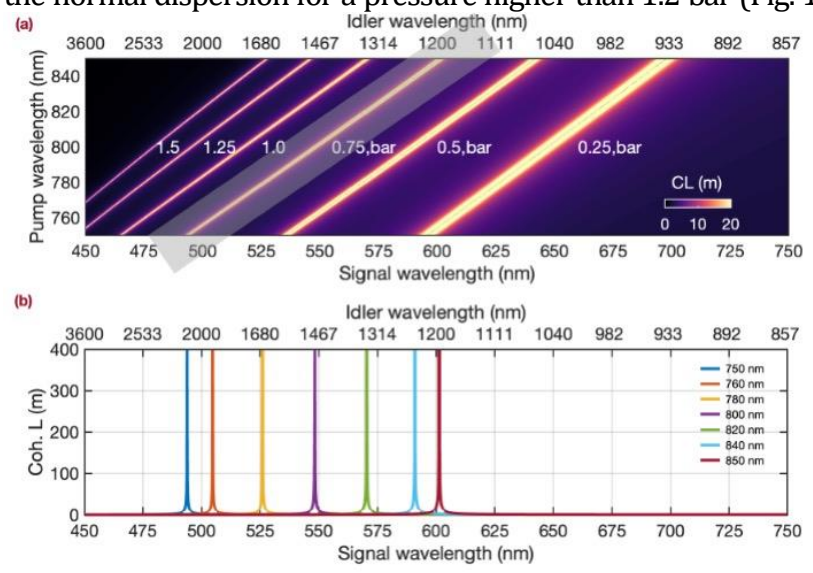

Fig. 2 a) Coherence length CL, for a tunable continuous pump wave at different gas pressures of Argon. The HCC has a core diameter of $150 \mu \mathrm{m}$. b) FWM band for a pressure of 0.75 bar and a tunable CW pump wave $(750-850 \mathrm{~nm})$.

For the ultra-fast FWM, the situation is more complicated. For a Fourier transform limited pump pulse, all the FWM bands temporally overlap within the walk-off length. However, in all cases, the pump owns a chirp since the pulse propagates in a weakly dispersive nonlinear medium creating a self-phase modulated spectrum. Alternatively, the pump chirp can be set at the input of the HCC to decrease the power. In both cases, the FWM bands generated from the chirped pump pulse were temporally delayed inducing a spectro-temporal distribution [29]. 


\section{SIMULATIONS AND DISCUSSION}

To explore accurately the role of the parameters on the parametric conversion and spectral bands, numerical simulations were performed by integrating the generalized nonlinear Schrödinger equation (NLSE) with the split-step Fourier method.

$\frac{\partial \hat{A}}{\partial z}-i\left(\beta(\Omega)-\beta_{0}-\beta_{1} \cdot \Omega\right) \hat{A}=i\left[\frac{\omega_{p} n_{2}}{A_{\text {eff }}}\right]\left(1+\frac{\Omega}{\omega_{p}}\right) F\left(|A|^{2} A\right)(\Omega)(6)$

where F stands for the Fourier transform.

In the following configuration, we use $2^{15}$ points and a temporal resolution of $1.3 \mathrm{fs}$. This led to a total window of $\sim \pm 21$ ps and a frequency span of $\sim \pm 374 \mathrm{THz}$. Before performing the numerical simulations, we checked the analytical calculation to ensure that the high order modes do not contribute to the generation of FWM in our configuration. Therefore, we only considered the propagation of the fundamental mode. $T$ is the time in a reference frame moving at the pump group velocity. $\tilde{A}$ is the initial total complex electric field in the frequency domain at the angular frequency $\Omega$. $\beta$ was calculated from Equation (5) and $\beta_{0}, \beta_{1}$ correspond to the zero and first coefficients of the Taylor expansion at the pump frequency. As the FWM process was investigated in an atomic gas, no delayed Raman effect was included in Eq. (6). The injected pump pulse has a central wavelength of $800 \mathrm{~nm}\left(\omega_{\mathrm{p}}=375 \mathrm{THz}\right)$ with a duration of $\sim 120 \mathrm{fs}$ at Full-Width at Half Maximum (FWHM) at the Fourier transform limit. The pump energy is $200 \mu \mathrm{J}$ leading to a peak power of $\sim 1.6 \mathrm{GW}$.

Fig. 3.a shows the evolution of the total simulated spectrum along a HCC for a 1.2 bar pressure and a total length of $60 \mathrm{~cm}$. The pulsed pump was injected with a random noise initial condition by adding half a photon per temporal mode. As expected, the spontaneous FWM side lobes became stronger during the propagation. The general shape of this band represents the gain spectrum in the unsaturation regime but it does not provide the absolute gain value since no input signal was injected. Selected output spectra are also shown in Figure. 3.b, for $\mathrm{L}=10 \mathrm{~cm}, 35 \mathrm{~cm}$, and $60 \mathrm{~cm}$. Two weak lobes appeared at a frequency offset of $\sim \pm 225 \mathrm{THz}$ from the pump that corresponds to $\sim 500$ and $\sim 2000 \mathrm{~nm}$. At the output, the bandwidth was wider in the anti-Stokes side as the consequence of the high order term [21,29]

For comparison, we calculated the gain profile from the analytical equations (Eq. 1-5) with a CW pump at $800 \mathrm{~nm}$. The spectral position of the FWM side lobes is in relatively good agreement with the pulsed case computed with Eq. 6 that takes into account the full bandwidth of the pump and other nonlinearities.

The main difference arouses in the instance of a broad pump pulse spectrum (pulsed case in the simulation), in which the spectrum bandwidth of the pump and FWM bands increased and were modulated. The modulations were mainly due to the self-phase modulation (SPM) process acquired on the pump pulse.

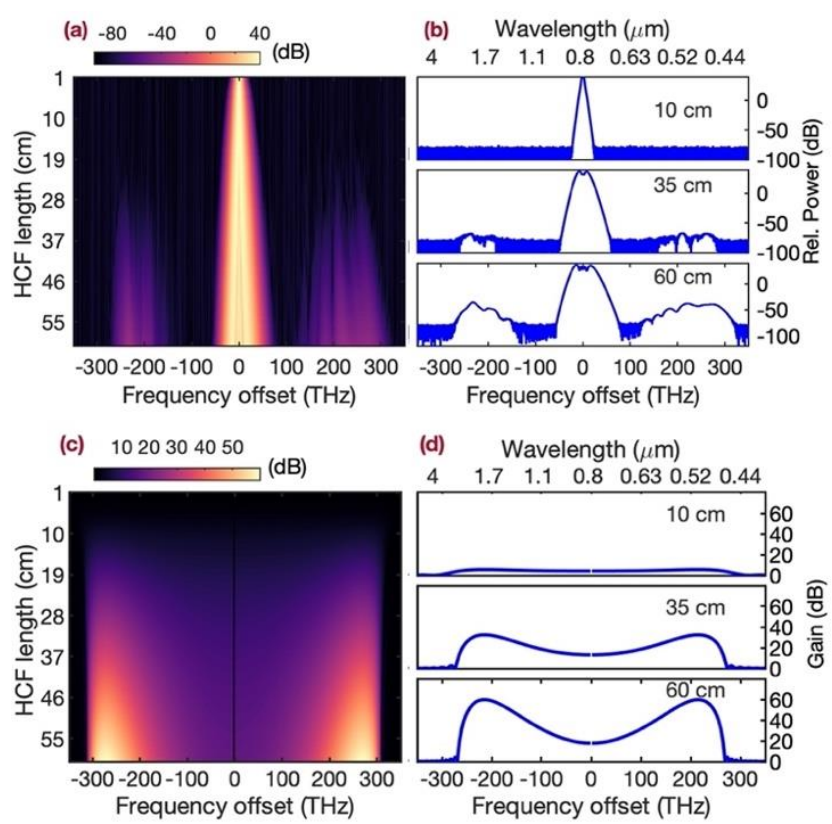

Fig. 3. $(a, c)$ Evolution of the spectrum along the HCC as the function of the frequency offset from the pump calculated from the NLSE with a pulsed pump and the analytical equations with a CW. (b, d) Selected spectrum at a length of 10,35 and $60 \mathrm{~cm}$. The pressure is 1.2 bar.

To limit the SPM contribution and increase the pulse energy, the pump pulse was stretched at the HCC input while maintaining a constant peak power. Fig. 4 shows the spectra when a positively chirped pulse (CP, grey curve) or a Fourier transform limited pulse (TLP, black curve) propagates in a $60 \mathrm{~cm}$ long HCC with a pressure of 1.2 bar.

The spectral and temporal profiles at $\mathrm{z}=60 \mathrm{~cm}$ are displayed in Fig. 4. $\mathrm{a}$ and $\mathrm{b}$. The chirped pulse was stretched to $200 \mathrm{fs}$ by applying a second order phase equals to $5807 \mathrm{fs}^{2}$. In both cases, the generation of the FWM bands clearly appeared at $\sim 30 \mathrm{~cm}$ (Fig. 4. $\mathrm{c}$ and e) and the side lobes were centered at a pump frequency offset of $\sim 225$ THz.

However, the spectral bands are strongly modulated for the case with the chirped pulse. It probably results from the interference between the FWM bands generated at different delays from the modulated pump spectrum. From the spectrogram displaying the spectral evolution with time (Fig. $4 \mathrm{~d}$ and f), it can be noted that each instantaneous pump frequency generates a well-defined band at a specific time. In both cases, the two spectral bands are asymmetric and the bandwidth is wider in the anti-Stokes side.

From the earlier description of the spontaneous FWM, several features were obtained, such as the center of the sidebands and their bandwidths. The next step is to include a broadband signal to either amplify an ultra-short pulse or to generate a tunable infrared idler pulse from the visible signal. Consequently, a signal pulse is now injected with a central wavelength of $530 \mathrm{~nm}$ to generate an idler at $1630 \mathrm{~nm}$. 


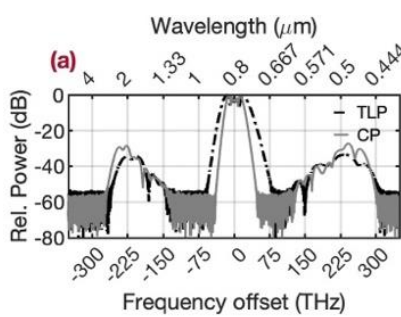

(b)
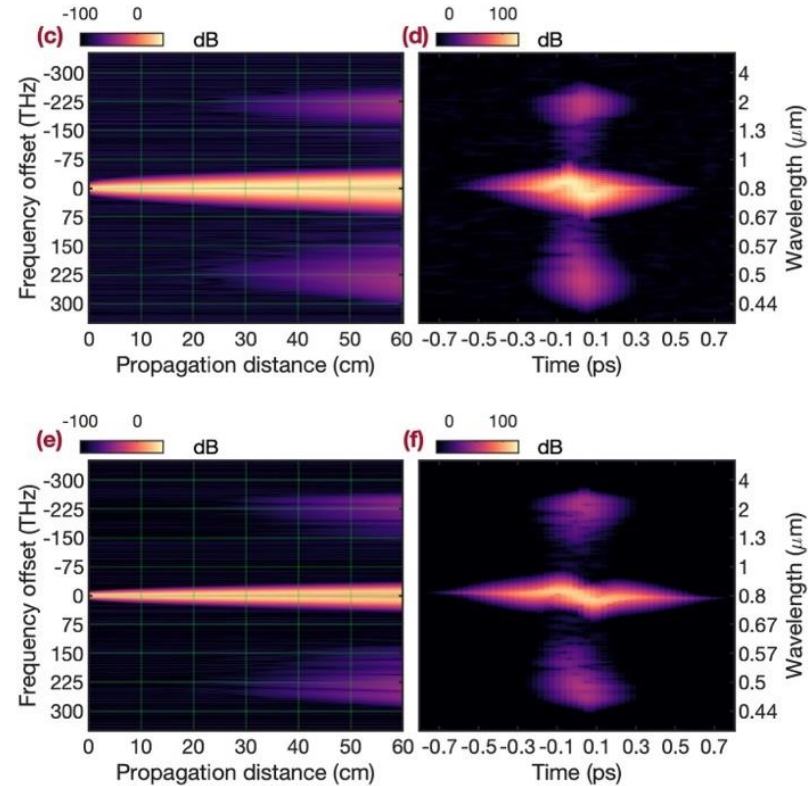

(f) $\stackrel{0 \quad 100}{\square}$

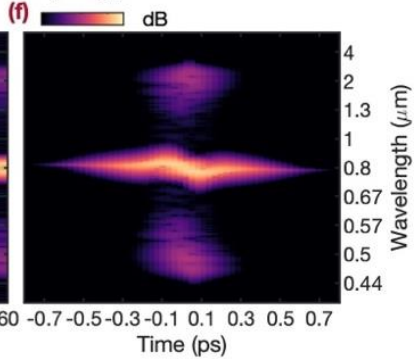

Fig. 4. a-b) Output spectral and temporal profiles at a length of $60 \mathrm{~cm}$ when the pump pulse is at the Fourier transform limit (FLP, black line) and chirped (CP, grey line) for a constant peak power. The pressure is $1.2 \mathrm{bar}$.

Spectrum as the function of the HCC length when the pump pulse is unchirped (c) and chirped (e). Corresponding spectrograms at $\mathrm{L}=60 \mathrm{~cm}$ are shown in (d) and (f).

The spectral bandwidth of the signal is $82 \mathrm{~nm}$ at FWHM (Figure 5.a). Its peak power is set at $264 \mathrm{~W}$. A number of configurations are reviewed and summarized in Table 1.

Table 1. Second order spectral phases and pulse durations of the pump and signal for the cases under study

\begin{tabular}{|l|l|l|l|}
\hline & $\begin{array}{l}\Phi_{\mathrm{p}}=0 \mathrm{fs}^{2} \\
\mathrm{~T}_{\mathrm{p}}=120 \mathrm{fs}\end{array}$ & $\begin{array}{l}\Phi_{\mathrm{p}}=5807 \mathrm{fs}^{2} \\
\mathrm{~T}_{\mathrm{p}}=200 \mathrm{fs}\end{array}$ & $\begin{array}{l}\Phi_{\mathrm{p}}=16524 \mathrm{fs}^{2} \\
\mathrm{~T}_{\mathrm{p}}=400 \mathrm{fs}\end{array}$ \\
\hline $\begin{array}{l}\Phi_{\mathrm{s}}=97 \mathrm{fs}^{2} ; \\
\mathrm{T}_{\mathrm{s}}=81 \mathrm{fs}\end{array}$ & Case I.a & & \\
\hline $\begin{array}{l}\Phi_{\mathrm{s}}=159 \mathrm{fs}^{2} ; \\
\mathrm{T}_{\mathrm{s}}=132 \mathrm{fs}\end{array}$ & Case I.b & & \\
\hline $\begin{array}{l}\Phi_{\mathrm{s}}=227 \mathrm{fs}^{2} ; \\
\mathrm{T}_{\mathrm{s}}=188 \mathrm{fs}\end{array}$ & & Case II.a & \\
\hline $\begin{array}{l}\Phi_{\mathrm{s}}=480 \mathrm{fs}^{2} ; \\
\mathrm{T}_{\mathrm{s}}=395 \mathrm{fs}\end{array}$ & & & Case II.b \\
\hline
\end{tabular}

In case I.a and I.b, the signal pulse is positively chirped with a spectral phase equals to $\Phi_{\mathrm{s}}=97 \mathrm{fs}^{2}$ or $159 \mathrm{fs}^{2}$, respectively. In this configuration, the signal pulse is either shorter (81 fs) or longer (132 fs) than the Fourier transform limited pump pulse (Figure 5.b). In both cases, the peak power is identical. In case II, the configuration is such that the pump and signal pulses both temporally overlap in the HCC (Figure 5.c). Thus, the two pulses are linearly chirped to reach a similar pulse duration equals to $188 \mathrm{fs}$ (case II.a) or 395 fs (case II.b) .
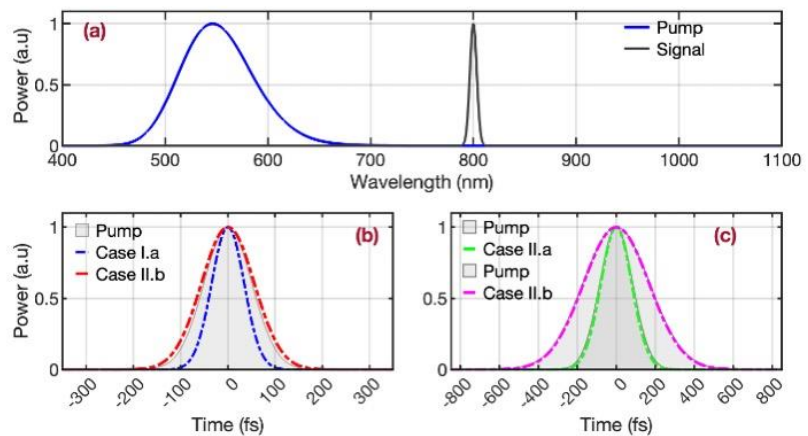

Fig. 5. (a) Normalized spectral intensity of the pump (black line), and signal (blue line) pulses b-c) Temporal profiles used in cases I and II.

Figure 6 shows the conversion efficiency, i.e $P_{s}(z=L) / P_{p}(0)$ and $\mathrm{P}_{\mathrm{i}}(\mathrm{z}=\mathrm{L}) / \mathrm{P}_{\mathrm{p}}(0)$, as the function of the pressure. For each case, the maximum efficiency is reached at a pressure between 1 and 1.25 bar and is higher in the idler side compared to the signal part. This optimal pressure range at this spectral range is expected from the calculated maximum CL (Figure 2.a). The conversion efficiency value is relatively high due to the injection of a strong signal power. It means that the process begins to deplete the pump pulses. To evaluate this effect, we plot the spectral and temporal profile evolutions as a function of the propagation length for the case I.b and II.a at 1 bar (Fig7.a and b). Clearly, the optical beating between the signal and the pump is observed, which lead to the FWM observed in the spectral domain. In the two cases, the FWM spectral bandwidth is wider in the Anti-Stokes side. For the case I.a and I.b, the conversion efficiency (Fig. 6.a) reached $\sim 8-12 \%$ for the signal or idler. The efficiency is slightly higher when the complete signal pulse interacts with the pump (case I.b).
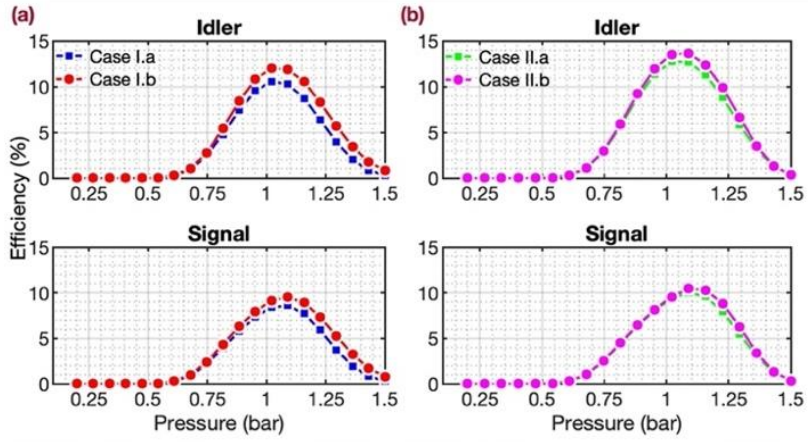

Fig. 6. (a) Signal and idler efficiency for case I, (b) Signal and idler efficiency for case II. 
(a)

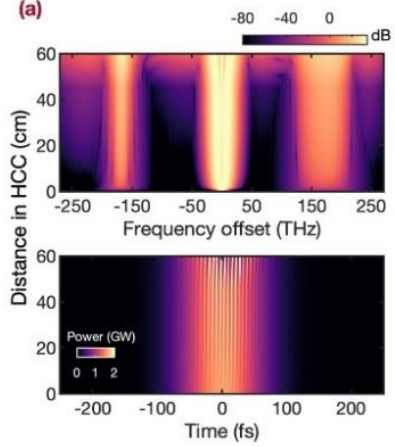

(b)
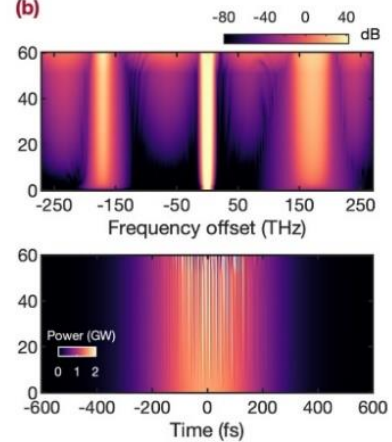

Fig.7 Spectral and temporal evolutions along the HCC, for case I.b (a) and II.a (b) at a pressure of 1.2 bar.

The spectral profile may also change with the delay, $\Delta \mathrm{T}$, between the pump and the injected signal. For example, Figure 8.a shows several output spectra in the case II.b when a relative delay of +0 or $+30,-70$ fs is added at the HCC input. While the signal and pump spectra are not significantly affected by the delay, the idler spectrum can be tuned. In addition to the GDM between the involved pulses, the chirps impact directly the instantaneous signal amplification and the idler generation. Since the signal is chirped, the lowest frequency is in the leading edge and the central wavelength (550 $\mathrm{nm}$ ) is centered at the null delay. However, at 1.2 bar the highest CL is achieved at $\sim 510 \mathrm{~nm}$ (Fig.2.a) and thus a negative delay improves the interaction of this spectral range with the maximum pump peak power (in the simplest case, set at a null delay). Moreover, as the exact value of the instantaneous pump frequency within the bandwidth influences the coherence length (Fig.2), the delay can also be adjusted to overlap together the optimal pump and signal spectral range. In our condition, the situation is more complex since the pump spectrum is also modulated due to the SPM effect and therefore, the maximum peak power is not necessarily centered to the central frequency of the pump spectrum. This behavior can also be explained through the spectrogram calculated at the HCC output (Fig. 8.b). For $\Delta \mathrm{T}=0$ fs and at the HCC output, the maximum of the FWM bands appear at $\sim 10$ fs from the center of the pump pulse where a portion of the pump pulse starts to deplete. When a delay is adjusted at the input, the overlap between the FWM bands and the pump occurs at another time. For $\Delta \mathrm{T}=+30 \mathrm{fs}$, the output FWM bands are marginally delayed and the idler generation is a slightly less efficient (Fig.8.a) since the signal pulse does not overlap with the maximum of the pump Gaussian profile at the HCC input. Alternatively, the temporal shift induced by the GDM can be compensated with a negative input delay. For $\Delta \mathrm{T}=-70 \mathrm{fs}$, the output pulses are centered at 0 fs; i.e., at the center of the pump pulse. It means that the signal and idler pulses overlap with the maximum of the pump peak power at the end of the HCC (Fig. 8.b) and start to deplete the center of the pump profile. In this case, the idler is generated further away from the pump, at $\sim 1.6 \mu \mathrm{m}$, since the chirped signal part is preferably amplified at $\sim 510 \mathrm{~nm}$ (Fig.8.a).
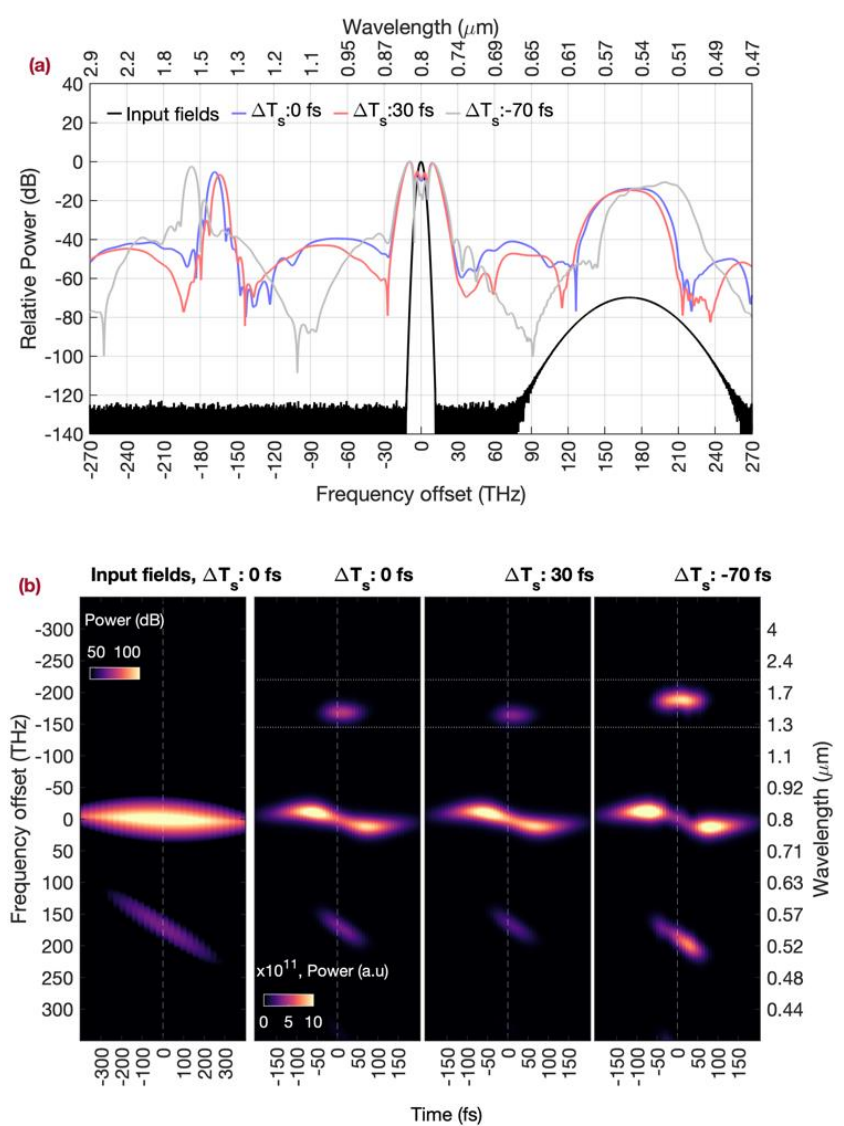

Fig. 8. (a) Output spectrum for selected delays between the pump and signal. (b) Spectrogram at the HCC input and output for selected delays. The input condition corresponds to Case II.b.

\section{EXPERIMENTAL IMPLEMENTATION}

In this section, we present an experimental implementation to highlight the generation and tunability of the infrared idler. Figure 9 shows the experimental setup. The HCC is located inside a metal chamber enclosed by two $5 \mathrm{~mm}$ thick quartz windows. A pressure manometer is attached to the chamber while the argon gas inflow is adjusted by input and output controllers.

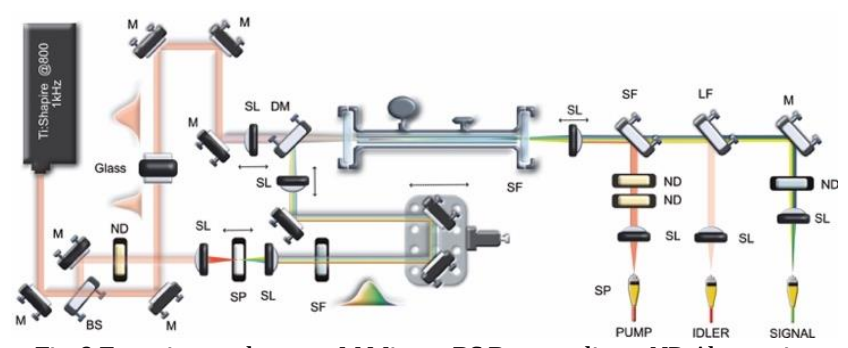

Fig. 9 Experimental set-up. M Mirror, BS Beam splitter, ND Absorptive neutral density filter, SL Spherical-plano Convex lens, DM Dichroic cold mirror, SF Sapphire plate, LF Long-pass filter, SF Short-pass filter, SP Spectrometer. 
The laser beam is generated from a Ti: Sapphire source emitting pulses at a central wavelength of $800 \mathrm{~nm}$ and a repetition rate of 1 $\mathrm{kHz}$. The pulse duration is $120 \mathrm{fs}$ at FWHM (Fig. 10). The beam is splitted into two paths with a $90 / 10$ beam splitter: the stronger part of the beam is used to pump the HCC, while the weaker part of the beam is utilized for the signal generation.

The signal is generated by focusing the weak beam $(\sim 5 \mu)$ into a 5$\mathrm{mm}$ thick sapphire plate to generate a filtered continuum whose spectral width extends from $450-600 \mathrm{~nm}$ (Fig. 11.a). A delay line is included in the signal path to ensure the temporal overlap between the pump and signal pulses. Then, the pump and signal beams are collinearly superposed with a cold mirror and focused with two plano-convex lenses towards the $30 \mathrm{~cm}$ long HCC with a core diameter of $150 \mu \mathrm{m}$. At the chamber output, the pump and signal energies were $\sim 170 \mu$ J and $0.539 \mathrm{~nJ}$, respectively.

At the input of the HCC, the pump pulse can also propagate through a dispersive block of N-F2 glass to stretch the pulse to 180 fs. For high pump power, the beam starts to self-focus in the dispersive block even if the pulse is stretched. Therefore, we fixed the peak power to $1.1 \mathrm{GW}$ for the stretched pulses and 1.6 GW for the unstretched pump. The autocorrelation traces of the pump are shown in Fig. 10.
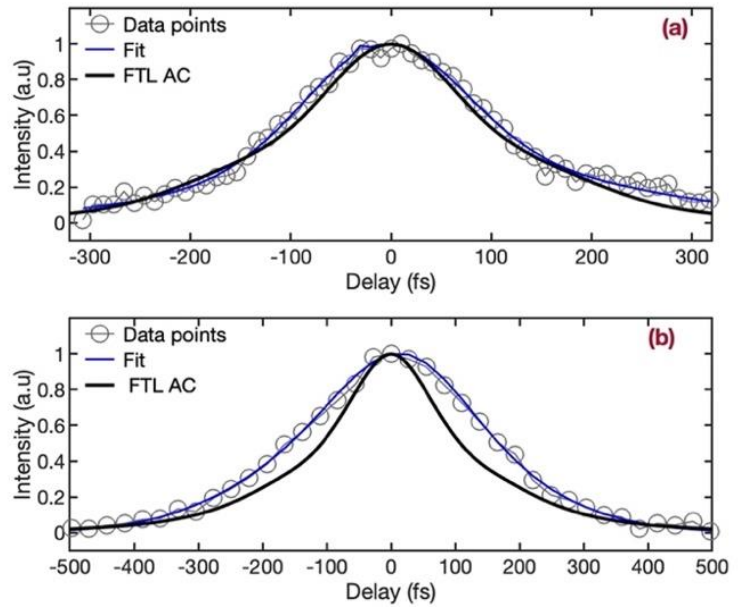

Fig.10. (a) Autocorrelation trace of the unchirped pump pulse (data points) with a Gaussian fit (blue lines), (b) Autocorrelation trace when the pump passes through a dispersive block of N-F2. The FTL AC (black curves) corresponds to the autocorrelation of the Fourier transform pulse calculated from the spectrum.

At the HCC output, the beam is split into three paths thanks to short and long-pass filters and the spectra are recorded simultaneously (pump, signal, and idler). The air was removed from the chamber, and subsequently filled with argon gas to 2 bar. Next, the pressure value was progressively reduced to 0.3 bar at which we observed the optimal generation of the idler in the stretched pump case. This is surprisingly different from the expected pressure ( 1.2 bar) obtained from the simulation. We believe this difference is due to other nonlinear effects. Particularly, high order modes are excited during the propagation. Therefore, the pump beam is also selffocused and spatial oscillations can occur during the propagation [30]. This might lead to local intensity increases and a change of the nonlinearity conditions.

As the continuum is also chirped from the nonlinear generation mechanism in the sapphire plate, the temporal overlap between the pump and the signal is adjusted for each pressure step to ensure the superposition of the specific spectral components to amplify with the pump pulse. For each step, the signal pump and idler properties were recorded with the same procedure.

Figure 11 shows an example of the signal, idler, and pump spectra recorded at the HCC output and with a specific delay of $19.2 \mathrm{fs}$. The signal is centered at $\sim 575 \mathrm{~nm}$ while the idler was generated at 1250 nm (Fig. 2).
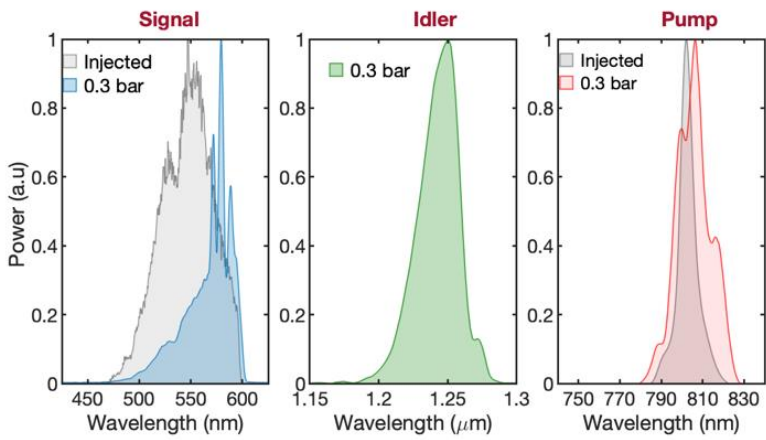

Fig. 11. Normalized spectrum of the input signal (grey line, the output signal (blue line), idler (green line), output pump for a pressure of $0.3 \mathrm{bar}$ (red line) or under vacuum (grey line).

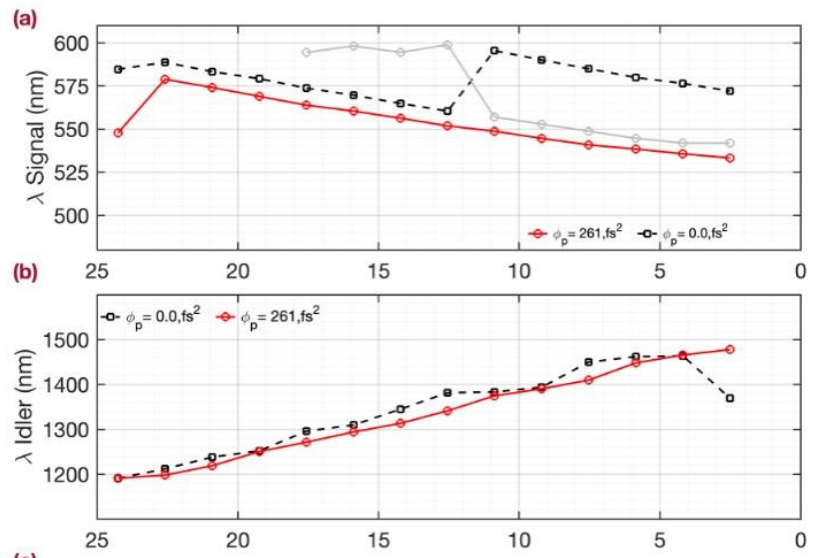

(c)

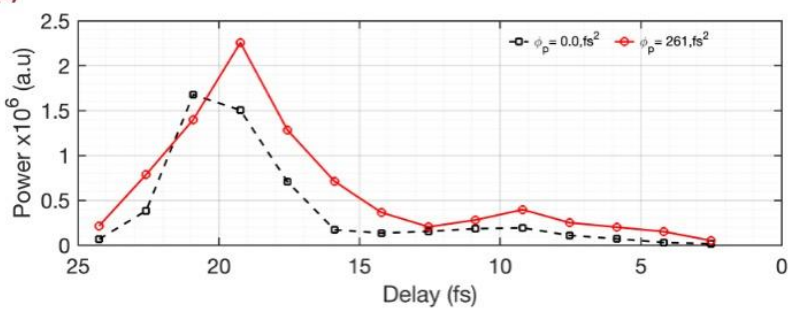

Fig. 12. (a-b) Maximum wavelength in the signal and idler output spectra as a function of the delay when the pump pulse is unstretched (black line) or stretched (red line). The gray line corresponds to a secondary maximum. c) Maximum power of the idler as a function of the delay.

As the delay is scanned, different parts of the chirped signal interact with the chirped pump pulse (see Fig. 8.b). Consequently, the maxima of the amplified signal and generated idler were reached at different wavelengths (Fig. 12.a and b) for each delay. By changing the delay, a tunable infrared idler pulse of 1.2 to $1.5 \mu \mathrm{m}$ was generated with a quasi-linear rate of $\sim 13.3 \mathrm{~nm} / \mathrm{fs}$ (Fig. 12. b) which depends on the signal chirp value. For the signal tunability (Fig. 12. a), the wavelength of maximum gain (red line) is linear with the delay, but on two distinct smaller zones. The same maximum can be 
obtained with two different delays. This is because the signal spectrum is strongly modulated when the pump pulse is chirped while the idler spectrum exhibits a single maximum (Fig. 13). This difference in the FWM band spectrum is also observed in the numerical simulations (Fig. 4.a).

As an example, the wavelength at the second maximum is also plotted (Fig. 12.a, grey line). The maximum power is reached at about $\sim 1250 \mathrm{~nm}$ (Fig. 12. c). We also reproduce the same experiment for the unchirped pump pulse (black curves). In this case, the wavelengths at maximum power change linearly with the delay (see Fig. 4.a) and the curve corresponds to one of the two preceding curves (Fig. 4.a red and gray curves).

At the HCC output, the idler (at $1.25 \mu \mathrm{m}$ ) pulses were measured using an intensity autocorrelator (Fig. 14). Assuming a Gaussian temporal profile, the pulse duration is $\sim 220 \mathrm{fs}$, at FWHM with pedestals at $\sim+/-600$ fs due to an uncompensated spectral phase while the FT limited pulse duration is $\sim 45$ fs thanks to the generation of a broad bandwidth in the infrared. The pulse energy at $1250 \mathrm{~nm}$ is relatively weak (few $\mathrm{n}$ ) and is consistent with the low gain value $(<5 \mathrm{~dB})$. Therefore, this scheme is suitable for use as a pre-amplifier to seed a subsequent ultra-fast amplifier. However, the low value can be significantly improved by increasing the HCC length to reach the multi- $\mu$ J level.

(a)

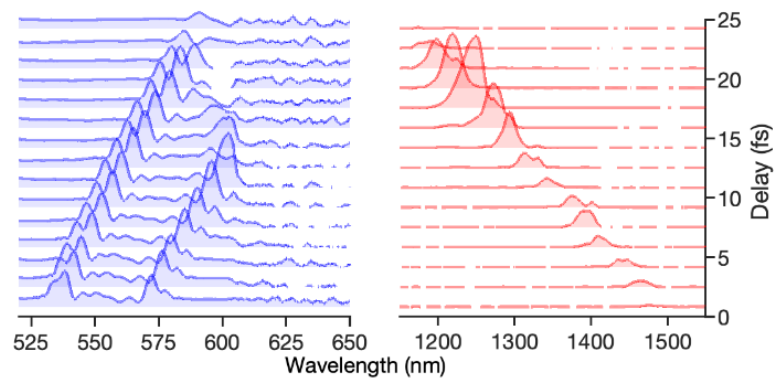

Fig. 13. Spectrum as the function of the delay for the signal (a) and idler (b) for a chirped pump pulse.

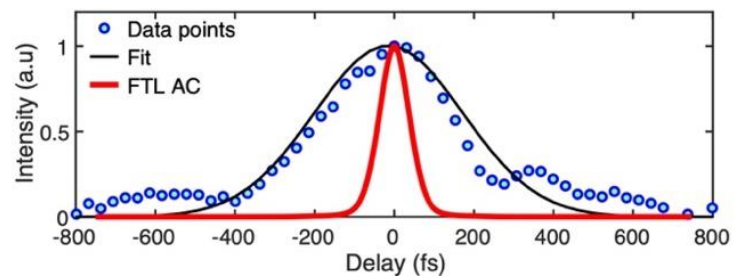

Fig. 14. Experimental autocorrelation trace of the idler (circles) and its Gaussian fit (black line). The red line corresponds to the FT limited pulse autocorrelation.

\section{CONCLUSIONS}

We investigated the tunability of infrared pulses generated by the FWM process in a gas filled HCC. We performed detailed analytical and numerical analyses of the process to understand the role of key parameters such as the gas pressure, the HCC length, GDM, pumpsignal frequency offset and delay, and the pump and signal chirps. At the HCC output, the femtosecond idler can be generated and tuned in the infrared region by controlling the pump-signal delay. An experiment was conducted to discuss the parameters and highlight the wide tunability from 1.2 to $1.5 \mu \mathrm{m}$. Ultra-short idler pulses are directly obtained at the HCC output and a duration of 45 fs can be reached if the phase is compensated. It is important to note that, the entire experiment was carried out with a $30 \mathrm{~cm}$ long HCC without the intention of optimizing conversion efficiency, but it can be implemented with a longer capillary (e.g. $60 \mathrm{~cm})$ to generate higher energy pulses. This configuration is very promising for the development of reliable, ultra-fast sources of tunable pulses in near and mid-infrared.

Funding. ANR (ANR-17-EURE-0002, ANR-10-IDEX-03-02), and Bourgogne Franche-Comté council (456789).

Acknowledgments. O. Zurita-Miranda benefits of a CONACyTGOBFrances (2018/710318). We also thank M.E. Ojo for helpful comments.

Disclosures. The authors declare no conflicts of interest.

Data Availability. Data underlying the results presented in this paper are not publicly available at this time but may be obtained from the authors upon reasonable request.

\section{References}

1. M. Musheghyan, P. P. Geetha, D. Faccialà, A. Pusala, G. Crippa, A. Campolo, A. G Ciriolo, M. Devetta, A. Assion, C. Manzoni, C. Vozzi, S. Stagira, "Tunable, few-cycle, CEP-stable mid-IR optical parametric amplifier for strong field applications," J. Phys. B: At. Mol. Opt. Phys. 53, 185402 (2020)

2. B. Shao,Y. Li, Y. Peng, P. Wang, J. Qian,Y. Leng,R. Li, "Broadbandwidth high-temporal-contrast carrier-envelope-phase-stabilized laser seed for 100 PW lasers," Opt. Lett. 45, 2215 (2020)

3. E. Rubino, J. Darginavičius, D. Faccio, P. Di Trapani, A. Piskarskas, A. Dubietis, "Generation of broadly tunable sub-30-fs infrared pulses by four-wave optical parametric amplification," Opt. Lett. 36, 382384 (2011)

4. J. Bromage, S.-W. Bahk, I. A. Begishev, C. Dorrer, M. J. Guardalben, B. N. Hoffman, J. B. Oliver, R. G. Roides, E. M. Schiesser, M. J. Shoup III, M. Spilatro, B. Webb, D. Weiner, J. D. Zuegel, "Technology development for ultra-intense all-OPCPA systems," High Power Laser Science and Engineering, 7, 1-11 (2019)

5. D. Bigourd, L. Lago, A. Mussot, A. Kudlinski, J.F. Gleyze, E. Hugonnot, "High gain fiber optical parametric chirped pulse amplification of femtosecond pulses at $1 \mu \mathrm{m}$," Opt. Lett. 35, 3480 (2010)

6. P. Morin, J. Dubertrand, P. Beaure d'Augeres, Y. Quiquempois, G. Bouwmans, A. Mussot, E. Hugonnot, " $\mu J$-level Raman-assisted fiber optical parametric chirped-pulse amplification,” Opt. Lett. 43, 46834686 (2018)

7. D. Bigourd, P. Morin, J. Dubertrand, C. Fourcade-Dutin, H. Maillotte, Y. Quiquempois, G. Bouwmans, E. Hugonnot, "Parametric gain shaping from a structured pump pulse," IEEE J. Phot. Tech. Lett. 31, 214 (2019)

8. D. Bigourd, P. d'Augerès, J. Dubertrand, E. Hugonnot, A. Mussot, "Ultra-broadband fiber optical parametric amplifier pumped by chirped pulses," Opt. Lett. 39, 3782 (2014)

9. C. Fourcade-Dutin, O. Vanvincq, A. Mussot, E. Hugonnot, D. Bigourd, "Ultra-broad band fiber optical parametric amplifier pumped by chirped pulses Part II : Sub-30 fs pulse amplification at high gain,” J. Opt. Soc. Am. B 32, 1488 (2015) 
10. W. Fu, F.W. Wise, "Normal-dispersion fiber optical parametric chirped-pulse amplification,” Opt. Lett. 43, 5331-5334 (2018)

11. A. G Ciriolo , A. Pusala, M. Negro, M. Devetta , D. Faccialà , G. Mariani, C. Vozzi, S. Stagira, "Generation of ultrashort pulses by four wave mixing in a gas-filled hollow core fiber," J. Opt. 20, 25503 (2018)

12. F. Belli, A. Abdolvand, J. C. Travers, P. J. Russell, "Highly efficient deep UV generation by four-wave mixing in gas-filled hollow-core photonic crystal fiber," Opt. Lett. 44, 5509-5512 (2019)

13. C. Durfee III, S. Backus, H. Kapteyn, M. Murnane, "Intense 8 fs pulse generation in the deep ultraviolet," Opt. Lett. 24, 697 (1999)

14. D. Faccio, A. Grün, P. K. Bates, O. Chalus, J. Biegert, "Optical amplification in the near-infrared in gas-filled hollow-core fibers," Opt. Lett. 34, 19, 2918 (2009)

15. Y. Kida, T. Imasaka, "Optical parametric amplification of a supercontinuum in a gas," App. Phys. B. 116, 673-680 (2014)

16. C. Fourcade-Dutin, O. Zurita-Miranda, P. Mounaix, D. Bigourd "Generation of ultra-short pulse in the mid-infrared in a hollow-core fiber," Fibers 9, 21 (2021)

17. S. O. Konorov, A. B. Fedotov, A. M. Zheltikov, "Enhanced fourwave mixing in a hollow-core photonic-crystal fiber," Opt. Lett. 28, 1448 (2003)

18. Xiaoyue Ding, Md. Selim Habib, R. Amezcua-Correa, and J. Moses, "Near-octave intense mid-infrared by adiabatic down-conversion in hollow anti-resonant fiber," Opt. Lett. 44, 1084-1087 (2019)

19. M. A. Finger, N. Y. Joly, P. St. J. Russell, M. V. Chekhova, "Characterization and shaping of the time-frequency Schmidt mode spectrum of bright twin beams generated in gas-filled hollow-core photonic crystal fibers," Phys. Rev. A 95053814 (2017)

20. M. Cordier, A. Orieux, B. Debord, F. Gérome, A. Gorse, M. Chafer, E. Diamanti, P. Delaye, F. Benabid, I. Zaquine, "Active engineering of fourwave mixing spectral correlations in multiband hollow-core fibers," Opt. Express 27, 9803-9814 (2019)

21. O. Vanvincq, C. Fourcade-Dutin, A. Mussot, E. Hugonnot, D. Bigourd, "Ultrabroadband fiber optical parametric amplifiers pumped by chirped pulses. Part 1: analytical model," J. Opt. Soc. Am. B 32,1479-1487 (2015)

22. M. E. Marhic, "Fiber Optical Parametric Amplifiers, Oscillators and Related Devices" (Cambridge University Press, 2008)

23. J. Hansryd, P. A. Andrekson, M. Westlund, J. Li, P.O. Hedekvist, "Fiberbased optical parametric amplifiers and their applications," IEEE J. Sel. Top. Quant. Electron. 8, 506-520 (2002)

24. A. Börzsönyi, Z. Heiner, M. P. Kalashnikov, A. P. Kovács, and K. Osvay, "Dispersion measurement of inert gases and gas mixtures at $800 \mathrm{~nm}$," Appl. Opt. 47, 4856-4863 (2008)

25. E. Marcatilli, R. Schmeltzer, "Hollow Metallic and Dielectric Waveguides for Long Distance Optical Transmission and Lasers", Bell Syst. Tech. J. 43, 1783-1809, (1964)

26. J. C. Travers, W. Chang, J. Nold, N. Y. Joly, and P. St. Russell, "Ultrafast nonlinear optics in gas-filled hollow-core photonic crystal fibers [Invited]," J. Opt. Soc. Am. B 28, A11-A26 (2011)

27. J. D. Harvey, R. Leonhardt, S. Coen, G. K. L. Wong, J. C. Knight, W. J. Wadsworth, P. St. J. Russell, "Scalar modulation instability in the normal dispersion regime by use of a photonic crystal fiber," Opt. Lett. 28, 2225 (2003)

28. C. Fourcade-Dutin, A. Imperio, R. Jamier, H. Muñoz, P. Perez Milan, H. Maillotte, P. Roy, D. Bigourd, "Temporal distribution of the parametric spectral gain in a photonic crystal fiber pumped by chirped pulses," Photonics 6, 20 (2019)

29. C. Fourcade-Dutin, D. Bigourd, "Modulation Instability in a dispersion oscillating fiber pumped by a broad band pulse," J. Modern Optics 64, 500-506 (2017)

30. G. Tempea, T. Brabec, "Theory of self-focusing in a hollow waveguide," Opt. Lett. 23, 762-764 (1998) 